ARTICLE

\title{
Self-amplifying RNA SARS-CoV-2 lipid nanoparticle vaccine candidate induces high neutralizing antibody titers in mice
}

Paul F. McKay (1) 1, Kai Hu', Anna K. Blakney (1) 1, Karnyart Samnuan', Jonathan C. Brown (1) 1, Rebecca Penn', Jie Zhou', Clément R. Bouton (1) 1, Paul Rogers (1) 1, Krunal Polra', Paulo J. C. Lin², Christopher Barbosa ${ }^{2}$, Ying K. Tam², Wendy S. Barclay ${ }^{1} \&$ Robin J. Shattock (iD ${ }^{1 凶}$

The spread of the SARS-CoV-2 into a global pandemic within a few months of onset motivates the development of a rapidly scalable vaccine. Here, we present a self-amplifying RNA encoding the SARS-CoV-2 spike protein encapsulated within a lipid nanoparticle (LNP) as a vaccine. We observe remarkably high and dose-dependent SARS-CoV-2 specific antibody titers in mouse sera, as well as robust neutralization of both a pseudo-virus and wild-type virus. Upon further characterization we find that the neutralization is proportional to the quantity of specific $\lg G$ and of higher magnitude than recovered COVID-19 patients. saRNA LNP immunizations induce a Th1-biased response in mice, and there is no antibodydependent enhancement (ADE) observed. Finally, we observe high cellular responses, as characterized by IFN- $\gamma$ production, upon re-stimulation with SARS-CoV- 2 peptides. These data provide insight into the vaccine design and evaluation of immunogenicity to enable rapid translation to the clinic.

\footnotetext{
${ }^{1}$ Department of Infectious Diseases, Imperial College London, Norfolk Place, London W2 1PG, UK. ${ }^{2}$ Acuitas Therapeutics, Vancouver, BC V6T 1Z3, Canada.

凶email: r.shattock@imperial.ac.uk
} 
T he unprecedented and rapid spread of SARS-CoV-2 into a global pandemic, with the current estimated number of confirmed cases $>8$ million people ${ }^{1}$, has motivated the need for a rapidly producible and scalable vaccine. Coronaviruses are positive-sense, single stranded RNA viruses that cause disease pathology ranging from the common cold to pneumonia ${ }^{2,3}$. Despite being listed on the WHO blueprint priority list, there are currently no licensed vaccines for SARS or MERS ${ }^{4}$. However, previous studies have elucidated the need to stabilize coronavirus spike proteins in their pre-fusion conformation in order to serve as a vaccine immunogen ${ }^{5}$.

Derived from an alphavirus genome $e^{6}$, self-amplifying RNA (saRNA) encodes the alphaviral replicase and a gene of interest (GOI), which enables replication of the RNA upon delivery to the cytoplasm. saRNA encapsulated in lipid nanoparticles (LNP) is a highly relevant platform for producing vaccines in the context of a global pandemic as it's possible to encode any antigen of interest ${ }^{7-9}$ and requires a minimal dose compared to messenger RNA (mRNA) ${ }^{10}$. The first RNA therapeutic, which is formulated in LNP, was approved in 2018 and has set the precedent for clinical safety of LNP-formulated RNA ${ }^{11}$.

Here, we compare the immunogenicity of saRNA encoding a pre-fusion stabilized SARS-CoV-2 spike protein encapsulated in LNP in a preclinical murine model to the immune response generated by a natural infection in recovered COVID-19 patients. We characterize both the humoral and cellular response as well as the neutralization capacity of a pseudotyped and wild-type SARSCoV-2 virus.

\section{Results}

saRNA LNPs induce high, Th-1 biased antibodies against SARS-CoV-2. After confirming expression of the pre-fusion stabilized SARS-CoV-2 spike protein in vitro (Supplementary Fig. 1), mice were immunized with saRNA encoding the SARSCoV-2 spike protein encapsulated in LNP with doses ranging from 0.01 to $10 \mu \mathrm{g}$ (Fig. 1a). Mice received two injections, one month apart, and electroporated plasmid DNA (pDNA) was used as a positive control while saRNA encoding the rabies glycoprotein $(\mathrm{RABV})$ in $\mathrm{pABOL}$ was used as a negative control. After 6 weeks, we observed remarkably high quantities of SARS-CoV-2 specific IgG in mouse sera in a dose-responsive manner, ranging from $10^{5}-10^{6} \mathrm{ng} \mathrm{mL}^{-1}$ (Fig. 1b). The groups that received doses of 10 and $1 \mu \mathrm{g}$ of saRNA LNP were significantly higher than the mice that received $10 \mu \mathrm{g}$ of electroporated $\mathrm{pDNA}$, with $p=0.0036$ and 0.0020 , respectively (two-way ANOVA adjusted for multiple comparisons). All of the saRNA LNP-vaccinated mice, even the $0.01 \mu \mathrm{g}$ group, had higher quantities of SARS-CoV-2 specific IgG compared to patients that had recovered from COVID-19, which had a mean titer of $10^{3} \mathrm{ng} \mathrm{mL}^{-1}$ and a range of $10^{1}-10^{5} \mathrm{ng} \mathrm{mL}^{-1}$. Importantly both the pDNA and saRNA LNP immunizations induced a Th1-biased response in mice (Fig. 2).

saRNA LNPs induce neutralization of pseudo- and wild-type SARS-CoV-2. We then sought to characterize how antibodies generated by immunization compared to those generated by a natural SARS-CoV-2 infection as far as capacity to neutralize both a SARS-CoV-2 pseudotyped and wild type virus (Fig. 1c, e). We observed highly efficient pseudoviral neutralization that varied in a linear dose-dependent manner for the mice vaccinated with saRNA LNP, with $\mathrm{IC}_{50}$ values ranging from $5 \times 10^{3}$ to $10^{5}$ and wild type viral neutralization titers of 80 to 20480 . The groups that received 10 or $1 \mu \mathrm{g}$ of saRNA LNP were significantly higher than the electroporated pDNA positive control group, both with $p<0.0001$ and RABV control for the wild type virus, with $p<0.0001$ and 0.0005 , respectively (two-way ANOVA adjusted for multiple comparisons). Comparison to the $\mathrm{IC}_{50}$ values of recovered COVID-19 patients, which had an average $\mathrm{IC}_{50}$ of $10^{3}$, revealed that even the lowest dose of saRNA LNP $(0.01 \mu \mathrm{g})$ in mice induced higher SARS-CoV-2 neutralization than a natural infection in humans.

SARS-CoV-2 IgG correlates directly with viral neutralization. We then determined if there is a correlation between the quantity of SARS-CoV-2 specific IgG and SARS-CoV-2 neutralization $\mathrm{IC}_{50}$ for both vaccinated mice and patients who have recovered from COVID-19. Both mice and patients have positive correlations between antibody level and viral neutralization, with $R^{2}=0.88$ and 0.87 and $p<0.0001$ and $=0.0007$ (linear regression), respectively, indicating that high antibody titers enable more efficient viral neutralization. Furthermore, we found that the SARS-CoV-2 specific IgG of LNP-vaccinated mice and recovered COVID-19 patients directly correlated with wild type virus neutralization titers, with $R^{2}=0.88$ and $p<0.0001$ and $R^{2}=0.93$ and $p=0.0019$ (linear regression), respectively. No antibodydependent enhancement (ADE) was observed after vaccination with saRNA LNP (Supplementary Fig. 2). We also tested the sera of vaccinated mice and recovered patients against other pseudotyped viruses, including SARS-CoV, MERS-CoV and 229E-CoV (Supplementary Fig. 3), and observed slight neutralization of SARS-CoV by vaccinated mice sera, but otherwise no crossreactivity.

Cellular and cytokine responses to saRNA LNP SARS-CoV-2 vaccine. We also characterized the cellular response and induction of systemic cytokines in response to vaccination with saRNA LNP (Fig. 3). We observed that splenocytes from vaccinated mice re-stimulated with a library of SARS-CoV-2 peptides yielded remarkably high IFN- $\gamma$ secretion as quantified by ELISpot (Fig. 3a). The saRNA LNP groups that received $0.01-10 \mu \mathrm{g}$ ranged from $1,000-2,600$ SFU per $10^{6}$ splenocytes, and the 1 and $10 \mu \mathrm{g}$ groups were significantly higher than the EP pDNA positive control group, with $p=0.0016$ and 0.0078 (Kruskal-Wallis test), respectively. The re-stimulated splenocyte secretions were also characterized with a panel of cytokines (Supplementary Fig. 4), with notable increases in GM-CSF, IL-10, IL-12, IL-17a, IL-21, IL-4, IL-5, IL-6, TNF- $\alpha$, IP-10, MIP- $1 \beta$ and RANTES.

We further characterized the immune response by assessing the systemic cytokine response $4 \mathrm{~h}$ after injection with saRNA LNP (Fig. 3b-g). The groups that received 10 and $1 \mu \mathrm{g}$ of saRNA LNP had enhanced levels of IL-6, MIP-1 $\beta$, RANTEs, IFN- $\beta$ and IP-10 in the sera compared to the RABV control group, indicating that the LNP formulation enables the immunogenicity of the saRNA. Data from the complete cytokine panel is presented in Supplementary Fig. 5.

\section{Discussion}

Here we characterized the immunogenicity of a SARS-CoV-2 saRNA LNP vaccine compared to the immune response of a natural infection in COVID-19 recovered patients. We observed that two saRNA LNP immunizations induced remarkably high SARS-CoV-2 specific IgG antibodies in mice, with quantities that were superior to both EP pDNA and natural infection in humans, that were able to efficiently neutralize a pseudotyped and wildtype virus. We also observed that the saRNA LNP vaccine induces a robust cellular response, which is partially enabled by the potent LNP formulation.

We observed that the saRNA-encoded pre-fusion stabilized spike protein of SARS-CoV-2 used in these studies is highly immunogenic, yielding antibody titers $>10^{6} \mathrm{ng} \mathrm{mL}^{-1}$ (Fig. 1), which is superior to what others have reported for subunit 
a

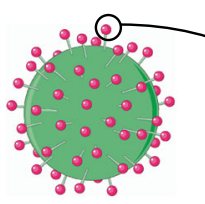

SARS-CoV-2

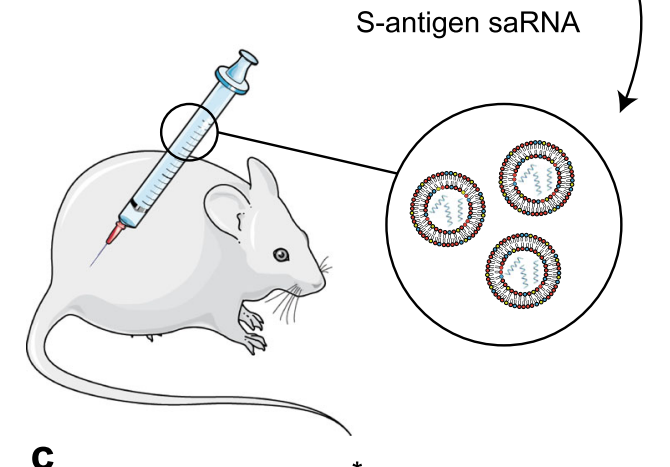

C

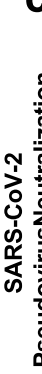

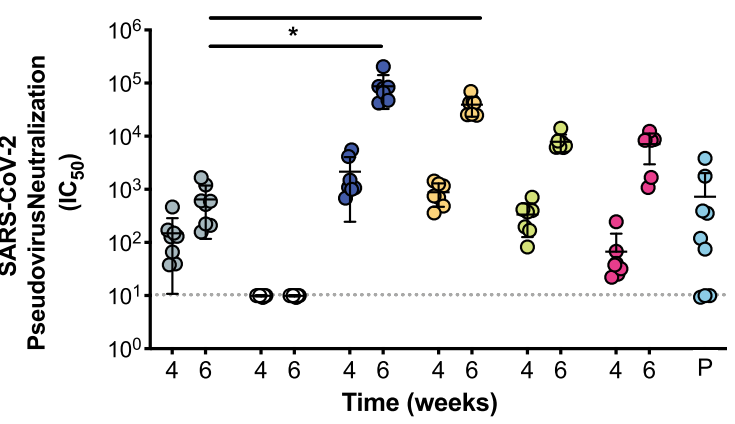

○ DNA + EP $\bigcirc$ RABV Control 0 LNP $10 \mu \mathrm{g}$

$\circ$ LNP $1 \mu \mathrm{g} \quad \mathrm{L}$ LNP $0.1 \mu \mathrm{g} \quad \circ$ LNP $0.01 \mu \mathrm{g}$

O Recovered COVID-19 Patients

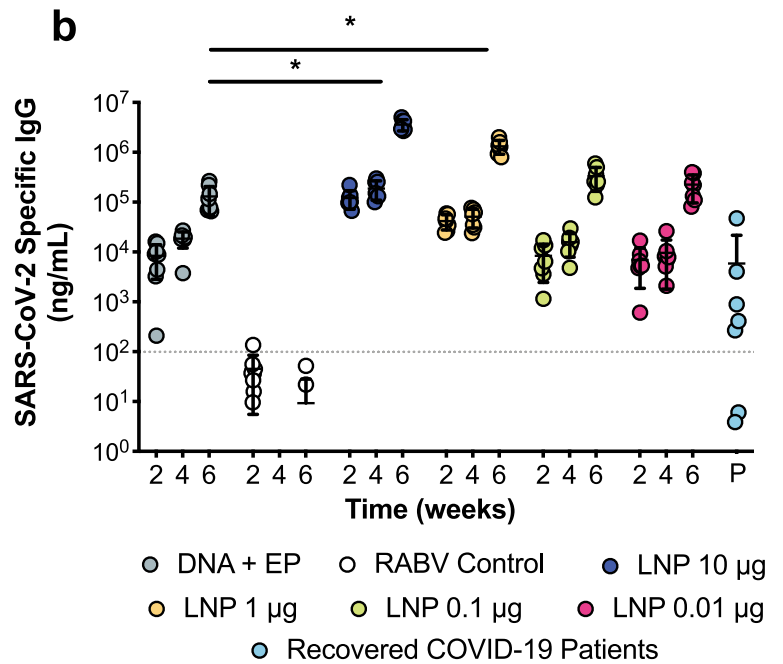

d

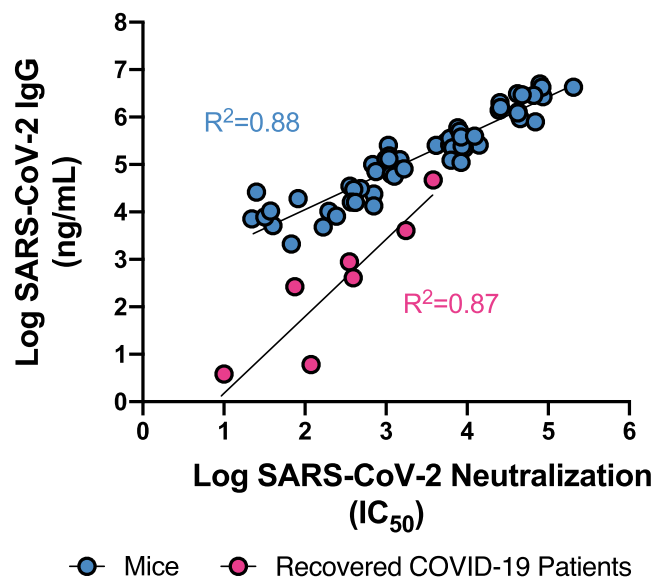

e

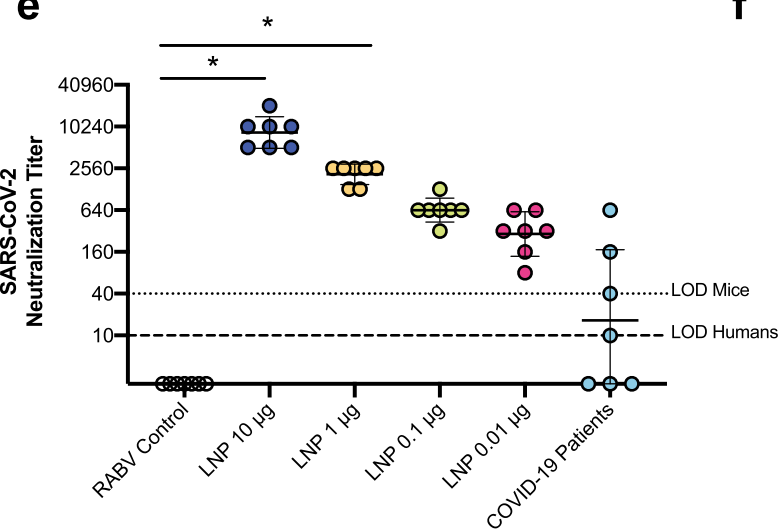

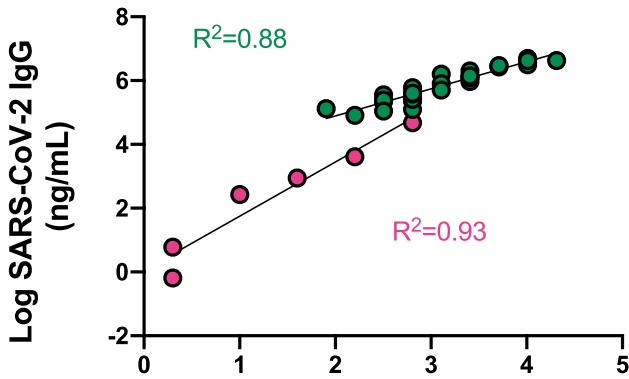

Log SARS-CoV-2 Neutralization Titer

- Mice o- Recovered COVID-19 Patients

Fig. 1 Antibody quantification and neutralization of a SARS-CoV-2 saRNA vaccinated mice compared to COVID-19 recovered patients. a Schematic of vaccination of $B A L B / c$ mice with saRNA encoding pre-fusion stabilized spike protein in LNP, $\mathbf{b}$ SARS-CoV-2 specific lgG responses in mice vaccinated with doses of LNP-formulated saRNA ranging from $0.01-10 \mu$ of saRNA with $n=7$ biologically independent animals and COVID-19 recovered patients with $n=9$ biologically independent samples, c SARS-CoV-2 pseudotyped virus neutralization of sera from BALB/c mice vaccinated with doses of LNPformulated saRNA ranging from 0.01-10 $\mu$ g of saRNA with $n=7$ biologically independent animals and COVID-19 recovered patients with $n=9$ biologically independent samples, $\mathbf{d}$ Correlation between SARS-CoV-2-specific IgG and SARS-CoV-2 neutralization IC 50 for vaccinated mice $(n=7$ biologically independent animals) and recovered COVID-19 patients ( $n=9$ biologically independent samples), e SARS-CoV-2 viral neutralization of sera from BALB/c mice vaccinated with doses of LNP-formulated saRNA ranging from 0.01 to $10 \mu \mathrm{g}$ of saRNA with $n=7$ biologically independent animals, $\mathbf{f}$ Correlation between SARS-CoV-2-specific IgG and SARS-CoV-2 wild type viral neutralization titers for vaccinated mice $(n=7$ biologically independent animals). Electroporated pDNA (DNA + EP) was used as a positive control while saRNA encoding the rabies glycoprotein (RABV) in pABOL was used as a negative control (RABV control). * indicates significance of $p<0.05$ using a two-way ANOVA adjusted for multiple comparisons. Line and error bars indicated mean \pm SD. Components of this figure were created using Servier Medical Art templates, which are licensed under a Creative Commons Attribution 3.0 Unported License; https://creativecommons.org/licenses/by/3.0/. 


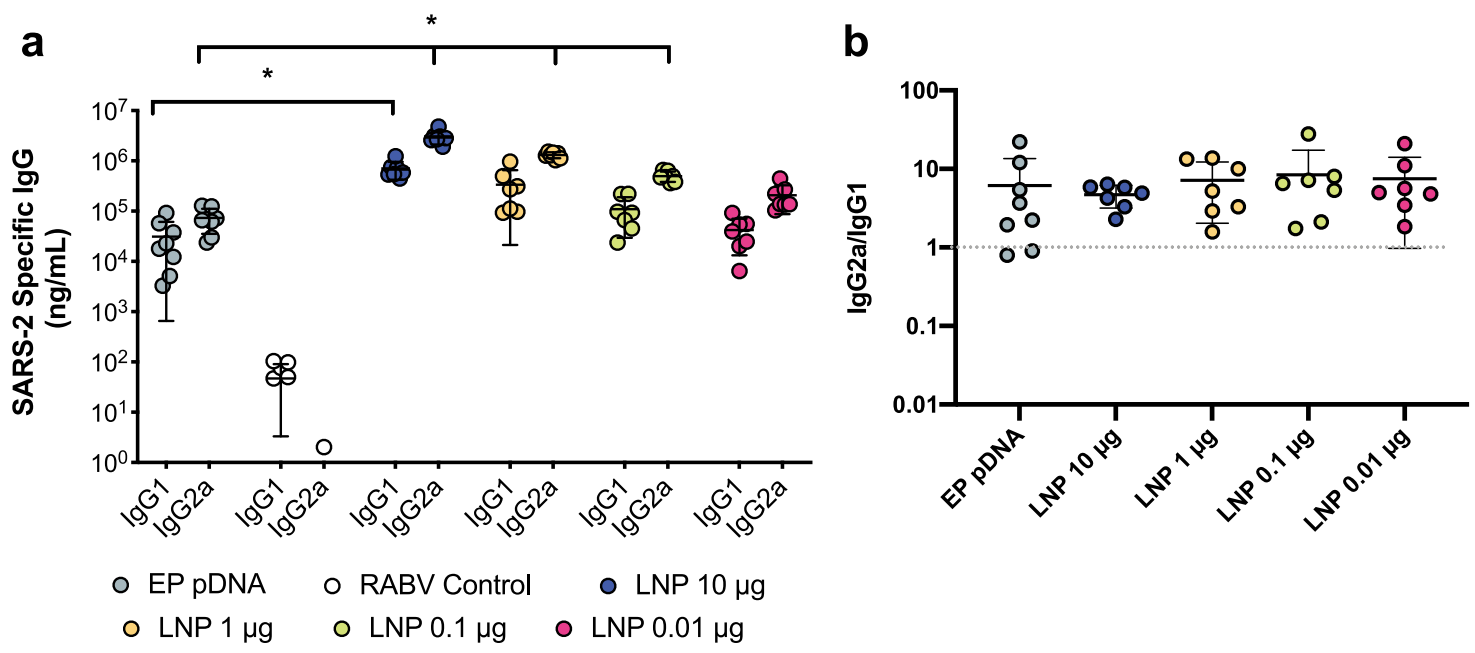

Fig. 2 Th1/Th2 skew in response to SARS-CoV-2 saRNA LNP vaccine. a lgG1 and lgG2a responses in mice vaccinated with doses of LNP-formulated saRNA ranging from $0.01-10 \mu \mathrm{g}$ of saRNA with $n=7$ biologically independent animals, $\mathbf{b}$ Th1/Th2 skewing responses in mice vaccinated with doses of LNP-formulated saRNA ranging from $0.01-10 \mu \mathrm{g}$ of saRNA with $n=7$ biologically independent animals, and $10 \mu \mathrm{g}$ of electroporated pDNA (EP pDNA) with $n=8$ biologically independent animals. The asterisk $\left(^{\star}\right)$ indicates significance of $p<0.05$ as determined by a Kruskal-Wallis test. Line and error bars indicated mean \pm SD.

vaccines for the SARS, MERS and SARS-2 coronaviruses ${ }^{12}$. Furthermore, we observed higher antibody titers, viral neutralization $\left(\mathrm{IC}_{50}\right)$ and cellular response for LNP-formulated saRNA than electroporated pDNA, which we postulate is due to the potent LNP used in these studies, as previous comparisons between polyplex-formulated saRNA and EP pDNA have yielded similar immunogenicity ${ }^{10}$. This is highly useful for translation as it means a potent LNP-formulated saRNA vaccine can be injected with a widely accepted syringe and needle, and does not require electroporation instrumentation, which we envision will enable more widespread vaccination to curb the spread of SARS-CoV-2.

The saRNA LNP vaccine presented in these studies elicited robust antibody and cellular responses, with a Th1 bias that we hypothesize will enable immunogenicity in humans. We did not observe any antibody-dependent enhancement (ADE) of SARSCoV-2 in our in vitro studies, as has been observed for SARS and MERS $^{13,14}$, but the role of this phenomena in vaccine-induced immunity is not yet fully understood. Overall, we believe that these data inform the antigen design, formulation and preclinical evaluation of immunogenicity that will enable rapid translation of a SARS-CoV-2 vaccine to the clinic trials.

\section{Methods}

Vectors. We used a plasmid vector to synthesize a self-amplifying RNA (saRNA) replicon, based on a Trinidad donkey Venezuelan equine encephalitis virus strain (VEEV) alphavirus genome (saRNA sequence is detailed in Supplementary Fig. 6, sequencing primers can be found in Supplementary Table 1). The viral structural proteins driven from the sub-genomic promoter were replaced by the surface 'spike' glycoprotein of the novel severe acute respiratory syndrome coronavirus 2 (SARS-CoV-2): GenBank accession number: QHD43416.1 with some modifications $^{15}$. The pre-fusion state of the spike glycoprotein was stabilized by proline substitutions of K968 and V969 ${ }^{16}$. We synthesized oligonucleotide fragments encoding the SARS-CoV-2 gene using GeneArt strings (Thermo Fisher Scientific) and assembled into the plasmid vector with NEB HiFi assembly (New England BioLabs). An expression plasmid expressing the same pre-fusion stabilized full length transmembrane protein used the pcDNA3.1 backbone and was directly synthesized and cloned into the vector by GeneArt (Thermo Fisher Scientific). A plasmid that expressed a soluble pre-fusion version was directly synthesized and cloned into the pcDNA3.1 backbone vector by GeneArt (Thermo Fisher Scientific). This soluble version ends at glutamine Q1208 of the pre-fusion modified QHD43416.1 gene sequence followed by a GGGGSGGGGS linker, a T4 fibritin (foldon) trimerization motif, a further GGGGSGGGGS linker, the Myc tag, a GSGSGS linker and finally an 8xHIS tag to enable purification of the soluble recombinant protein. The RABV control saRNA was based on the Pasteur strain: GenBank accession number: NP_056796.1 with the F318V amino acid substitution to reduce glycoprotein binding to the neurotrophin receptor (p75NTR), a natural ligand.

Recombinant soluble SARS-CoV-2 S expression and purification. The plasmid expressing the soluble pre-fusion version of SARS-CoV-2 S was used to produce the recombinant protein using the FreeStyle ${ }^{\mathrm{m}} \mathrm{2} 293$ Expression System (Thermo Fisher Scientific), according to the manufacturer's instructions. Conditioned medium was clarified by centrifugation and protein was sequentially purified by a HisTrap HP column and a HiPrep 16/60 Sephacryl S-300 HR size exclusion chromatography (SEC) column (both from GE Healthcare). Purified protein was first analyzed by Native-PAGE and Western blot, and then filtered through a $0.22 \mu \mathrm{m}$ membrane, aliquoted and stored at $-80^{\circ} \mathrm{C}$.

In vitro transcription of RNA. Self-amplifying RNA encoding the pre-fusion stabilized SARS-CoV-2 was produced using in vitro transcription. pDNA was transformed into E. coli (New England BioLabs, UK), cultured in $100 \mathrm{~mL}$ of Luria Broth (LB) with $100 \mu \mathrm{g} \mathrm{mL}^{-1}$ carbenicillin (Sigma Aldrich, UK). Plasmid was purified using a Plasmid Plus MaxiPrep kit (QIAGEN, UK) and the concentration and purity was measured on a NanoDrop One (ThermoFisher, UK). pDNA was linearized using MluI for $3 \mathrm{~h}$ at $37^{\circ} \mathrm{C}$. Uncapped in vitro RNA transcripts were produced using $1 \mu \mathrm{g}$ of linearized DNA template in a MEGAScript ${ }^{\mathrm{m}}$ reaction (Ambion, UK) for $2 \mathrm{~h}$ at $37^{\circ} \mathrm{C}$, according to the manufacturer's protocol. Transcripts were then purified by overnight $\mathrm{LiCl}$ precipitation at $-20^{\circ} \mathrm{C}$, centrifuged at $14,000 \mathrm{RPM}$ for $20 \mathrm{~min}$ at $4^{\circ} \mathrm{C}$ to pellet, washed with $70 \% \mathrm{EtOH}$, centrifuged at 14,000 RPM for 5 min at $4{ }^{\circ} \mathrm{C}$ and resuspended in UltraPure $\mathrm{H}_{2} \mathrm{O}$ (Ambion, UK). Purified transcripts were capped using the ScriptCap ${ }^{\mathrm{mm}}$ Cap 1 Capping System Kit (CellScript, WI, USA) for $2 \mathrm{~h}$ at $37^{\circ} \mathrm{C}$, according to the manufacturer's protocol. Capped transcripts were purified by $\mathrm{LiCl}$ precipitation as described above, resuspended in RNA storage buffer (10 mM HEPES, $0.1 \mathrm{mM}$ EDTA, and $100 \mathrm{mg} \mathrm{mL}^{-1}$ trehalose) and stored at $-80^{\circ} \mathrm{C}$ until further use.

Cell culture \& saRNA transfection. HEK293T/17 cells (ATCC) and Vero-E6 cells (African green monkey VERO C1008 [Vero 76, clone E6, Vero E6] (ATCC ${ }^{\circledR}$ CRL$\left.1586^{\mathrm{m}}\right)$ ) were cultured in complete Dulbecco's Modified Eagle's Medium (DMEM) (Gibco, Thermo Fisher Scientific) containing 10\% fetal bovine serum (FBS, Gibco, Thermo Fisher Scientific), 1\% L-glutamine and 1\% penicillin-streptomycin (Thermo Fisher Scientific). For Caco2 cells (ATCC) culture medium was modified to include $20 \%$ fetal bovine serum. All cells were cultured at $37^{\circ} \mathrm{C}, 5 \% \mathrm{CO}_{2}$. HEK293T/17 cells (ATCC) were plated in a 12-well plate at a density of $0.75 \times 10^{6}$ cells per well $48 \mathrm{~h}$ prior to transfection. Lipofectamine MessengerMAX (Thermo Fisher Scientific) was used according to the manufacturer's instructions for the transfection of SARS-CoV-2 saRNA.

Flow cytometry. Twenty-four hours post transfection, cells were harvested and resuspended in $1 \mathrm{~mL}$ of FACS buffer (PBS $+2.5 \%$ FBS) at a concentration of $1 \times$ $10^{7}$ cells $/ \mathrm{mL}$. One hundred microliters of the resuspended cells was added to a FACS tube and stained with $50 \mu \mathrm{L}$ of Live/Dead Fixable Aqua Dead Cell Stain (Catalog \#L34965, Thermo Fisher Scientific) at a 1:400 dilution on ice for $20 \mathrm{~min}$. Cells were then washed with $2.5 \mathrm{~mL}$ of FACS buffer and centrifuged at $1750 \mathrm{RPM}$ 
a
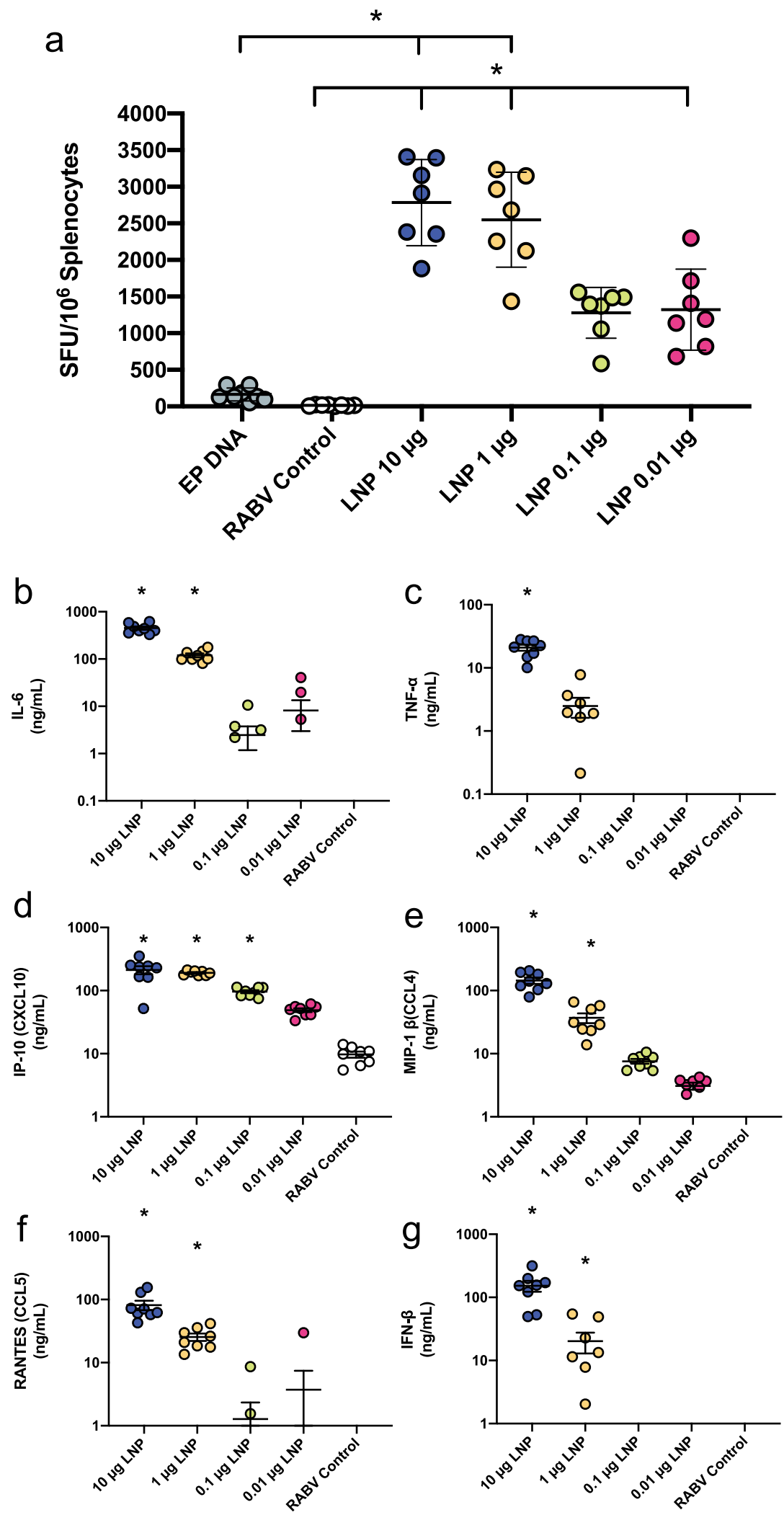

Fig. 3 Cellular and secreted cytokine responses to a SARS-CoV-2 saRNA LNP vaccine. a Quantification of IFN- $\gamma$ splenocytes upon restimulation with SARS-CoV-2 peptides, expressed as spot forming units (SFU) per $10^{6}$ cells with $n=7$ biologically independent animals. Electroporated pDNA (EP pDNA) was used as a positive control while saRNA encoding the rabies glycoprotein (RABV) in $\mathrm{pABOL}$ was used as a negative control (RABV control). b-g Cytokine profile in sera of mice $4 \mathrm{~h}$ after vaccination with SARS-CoV-2 LNP vaccine with $n=7$ biologically independent animals. Remaining cytokines can be found in Supplementary Fig. 5. The asterisk $\left(^{\star}\right)$ indicates significance of $p<0.05$ as determined by a Kruskal-Wallis test. Line and error bars indicated mean $\pm S D$. 
for $7 \mathrm{~min}$. After centrifugation, cells were stained with $1 \mu \mathrm{g}$ (1:25 dilution) of a SARS-CoV spike protein polyclonal antibody (Catalog \#PA1-41165, Thermo Fisher Scientific) for $30 \mathrm{~min}$ on ice before washing with $2.5 \mathrm{~mL}$ of FACS buffer and centrifuging at $1750 \mathrm{RPM}$ for $7 \mathrm{~min}$. Cells were then stained with $0.4 \mu \mathrm{g}(1: 62.5$ dilution) of FITC goat anti-rabbit IgG (Catalog \#554020, BD Pharmigen) for $30 \mathrm{~min}$ on ice. After incubation, cells were washed with $2.5 \mathrm{~mL}$ of FACS buffer, centrifuged at $1750 \mathrm{RPM}$ for $7 \mathrm{~min}$ and resuspended with $250 \mu \mathrm{L}$ of PBS. Cells were fixed with $250 \mu \mathrm{L}$ of $3 \%$ paraformaldehyde for a final concentration of $1.5 \%$. Samples were analyzed on a LSRFortessa (BD Biosciences) with FACSDiva software (BD Biosciences). Data were analyzed using FlowJo Version 10 (FlowJo LLC).

Formulation of saRNA. saRNA was encapsulated in LNP using a self-assembly process in which an aqueous solution of saRNA at $\mathrm{pH}=4.0$ is rapidly mixed with an ethanolic lipid mixture ${ }^{17}$. LNP used in this study were similar in composition to those described previously 18,19 , which contain an ionizable cationic lipid (proprietary to Acuitas)/phosphatidylcholine/cholesterol/PEG-lipid. The proprietary lipid and LNP composition are described in US patent US10,221,127. They had a mean hydrodynamic diameter of $\sim 75 \mathrm{~nm}$ with a polydispersity index of $<0.1$ as measured by dynamic light scattering using a Zetasizer Nano ZS (Malvern Instruments Ltd, Malvern, UK) instrument and an encapsulation efficiency of $>90 \%$.

RABV control group was formulated with $8 \mathrm{kDa} \mathrm{pABOL}$ at a ratio of polymer to RNA of 45:1 (w/w) using the titration method $^{8}$. RNA and pABOL were diluted in HEPES buffer (20 mM HEPES, 5 wt.\% glucose in water, $\mathrm{pH} 7.4)$ at a volume ratio of 4:1 (RNA solution to pABOL solution). The polymer solution was added to a centrifuge tube and place on a stir plate with a stir bar and mixed at 1200 RPM. The RNA solution was then added to the polymer solution at a flow rate of $160 \mu \mathrm{L} \mathrm{min}{ }^{-1}$.

Animals and immunizations. BALB/c mice aged 6-8 weeks old were placed into groups of $n=7$ or 8 . Animals were handled and procedures were performed in accordance with the terms of a project license granted under the UK Home Office Animals (Scientific Procedures) Act 1986. All the procedures and protocols used in this study were approved by an animal ethical committee, the Animal Welfare and Ethical Review Body (AWERB). Groups of mice were injected intramuscularly (IM; quadriceps) with a $50 \mu \mathrm{L}$ of vaccine saRNA formulations. For animals that were vaccinated with pDNA, $10 \mu \mathrm{g}$ of pDNA was injected in $50 \mu \mathrm{L}$ PBS followed by electroporation (EP) using 5-mm electrodes using an ECM 830 square-wave electroporation system (BTX) (pulses: $100 \mathrm{~V}$ of positive and negative polarity at 1 pulse $\mathrm{s}^{-1}, 50 \mathrm{~ms}$ pulse). Animals were immunized at week 0 , boosted with a second vaccination at week 4 and euthanized using a Schedule 1 method at week 6 , at which time the spleens were removed and processed to single cells for use in assays. Serum samples were collected at two-week intervals.

Recovered COVID-19 patient samples. Serum samples were donated to the Communicable Diseases Research Tissue Bank, Section of Virology, Imperial College London, following written informed consent, by patients who had been infected with SARS-CoV-2. The tissue bank is approved by the National Research Ethics Service, South Central Committee Oxford C (Ref 15/SC/0089). All patients with suspected COVID-19 were attended to at the St. Mary's Hospital in London, United Kingdom. All patients had previously tested PCR-positive during their hospital stay, but then were PCR-negative at the time of sampling.

IFN- $\gamma$ ELISpots. Assessment of the IFN- $\gamma$ T cell response was performed using the Mouse IFN- $\gamma$ ELISpot ${ }^{\text {PLUS }}$ kit (Mabtech) following the manufacturer's instructions. Briefly, anti-IFN- $\gamma$ pre-coated plates were blocked with DMEM $+10 \%$ FBS for at least $30 \mathrm{~min}$, then cells were added at $2.5 \times 10^{5}$ cells per well for negative control (media only) and SARS-CoV-2 peptide pools (15-mers overlapping by 11; JPT Peptides) $(1 \mu \mathrm{g} \mathrm{mL}-1)$ in $200 \mu \mathrm{L}$ final volume per well. The positive control wells contained $5 \times 10^{4}$ cells per well in $200 \mu \mathrm{L}$ final volume per well with $5 \mu \mathrm{g} \mathrm{mL}$ ${ }^{-1}$ of ConA. Plates were incubated overnight at $5 \% \mathrm{CO} 2,37^{\circ} \mathrm{C}$ incubator and developed as per the manufacturer's protocol. Once dried, plates were read using the AID ELISpot reader ELR03 and AID ELISpot READER software (Autoimmun Diagnostika GmbH).

Antigen-specific IgG ELISA. The antigen-specific IgG, IgG1 and IgG2a titres in mouse sera were assessed by a semi-quantative ELISA ${ }^{20}$. MaxiSorp high binding ELISA plates (Nunc) were coated with $100 \mu \mathrm{L}$ per well of $1 \mu \mathrm{g} \mathrm{mL}^{-1}$ recombinant SARS-CoV-2 protein with the pre-fusion stabilized conformation in PBS. For the standard IgG/IgG1/IgG2a, 3 columns on each plate were coated with 1:1000 dilution each of goat anti-mouse Kappa (Catalog \#1050-01, Southern Biotech) and Lambda light chains (Catalog \#1060-01, Southern Biotech). After overnight incubation at $4^{\circ} \mathrm{C}$, the plates were washed 4 times with PBS-Tween $200.05 \%(\mathrm{v} / \mathrm{v})$ and blocked for $1 \mathrm{~h}$ at $37^{\circ} \mathrm{C}$ with $200 \mu \mathrm{L}$ per well blocking buffer (1\% BSA (w/v) in PBS-Tween- $200.05 \%(\mathrm{v} /$ $\mathrm{v})$ ). The plates were then washed and the diluted samples or a 5 -fold dilution series of the standard IgG (or IgG1 or IgG2) added using $50 \mu \mathrm{L}$ per well volume. Plates were incubated for $1 \mathrm{~h}$ at $37^{\circ} \mathrm{C}$, then washed and secondary antibody added at 1:2000 dilution in blocking buffer ( $100 \mu \mathrm{L}$ per well) using either anti-mouse IgG-HRP (Catalog \#1030-05, Southern Biotech), anti-mouse IgG1-HRP (Catalog \#1070-05,
Southern Biotech) or anti-mouse IgG2a-HRP (Catalog \#1080-05, Southern Biotech). After incubation and washes, plates were developed using $50 \mu \mathrm{L}$ per well SureBlue $\operatorname{TMB}\left(3,3^{\prime}, 5,5^{\prime}\right.$-tetramethylbenzidine) substrate and the reaction stopped after $5 \mathrm{~min}$ with $50 \mu \mathrm{L}$ per well stop solution (Insight Biotechnologies). The absorbance was read on a Versamax Spectrophotometer at $450 \mathrm{~nm}$ (BioTek Industries).

Pseudotype virus neutralization assay. A HIV-pseudotyped luciferase-reporter based system was used to assess the neutralization ability of sera from vaccinated animals and recovered patients against SARS-CoV, SARS-CoV-2, MERS-CoV and $229 \mathrm{E}-\mathrm{CoV}^{21,22}$. In brief, CoV S-pseudotyped viruses were produced by cotransfection of 293T/17 cells with a HIV-1 gag-pol plasmid (pCMV- 48.91 , a kind gift from Prof. Julian Ma, St George's University of London), a firefly luciferase reporter plasmid (pCSFLW, a kind gift from Prof. Julian Ma, St George's University of London) and a plasmid encoding the $\mathrm{S}$ protein of interest (pSARS-CoV-S, pSARS-CoV2-S, pMERS-CoV-S or p229E-CoV-S) at a ratio of 1:1.5:1. Viruscontaining medium was clarified by centrifugation and filtered through a $0.45 \mu \mathrm{m}$ membrane $72 \mathrm{~h}$ after transfection, and subsequently aliquoted and stored at $-80^{\circ} \mathrm{C}$. For the neutralization assay, heat-inactivated sera were first serially diluted and incubated with virus for $1 \mathrm{~h}$, and then the serum-virus mixture was transferred into wells pre-seeded Caco2 cells. After $48 \mathrm{~h}$, cells were lysed, and luciferase activity was measured using Bright-Glo Luciferase Assay System (Promega). The $\mathrm{IC}_{50}$ neutralization was then calculated using GraphPad Prism (version 8.4). We analyzed the pseudovirus neutralization data titration curves looking for any antibodydependent enhancement (ADE) of infection, to determine if we could observe an altered elevated baseline level in the antibody treated wells when compared to either the control or virus alone conditions.

Wild-type viral neutralization assay. The ability of sera from vaccinated animals to neutralize wild type SARS-CoV-2 virus was assessed by neutralization assay on Vero-E6 cells. SARS-CoV-2/England/IC19/2020 was isolated on Caco2 cells from a clinical sample collected from a patient admitted to St. Mary's Hospital in London, United Kingdom. Heat-inactivated sera were serially diluted in assay diluent consisting of DMEM (Gibco, Thermo Fisher Scientific) with 1\% penicillinstreptomycin (Thermo Fisher Scientific), 0.3\% BSA fraction V (Thermo Fisher Scientific) and $0.25 \mu \mathrm{g} \mathrm{mL}^{-1}$ TPCK trypsin (Worthington). Serum dilutions were incubated with 100 TCID50 per well of SARS-CoV-2/England/IC19/2020 diluted in assay diluent for $1 \mathrm{~h}$ at RT and transferred to 96-well plates pre-seeded with Vero-E6 cells. Serum dilutions were performed in duplicate. Plates were incubated at $37^{\circ} \mathrm{C}, 5 \% \mathrm{CO}_{2}$ for 5 days before adding an equal volume of $2 \mathrm{X}$ crystal violet stain to wells for $1 \mathrm{~h}$. Plates were washed, wells were scored for cytopathic effect and a neutralization titer calculated as the reciprocal of the highest serum dilution at which full virus neutralization occurred.

Cytokine measurement in splenocytes and sera. Splenoctyes isolated from each individual mouse were plated into round bottom 96 well plates $\left(1 \times 10^{6}\right.$ per well in a $200 \mathrm{uL}$ total volume) and cultured for 7 days with media alone, $5 \mu \mathrm{g} \mathrm{mL}{ }^{-1}$ SARS$\mathrm{CoV}-2$ recombinant protein or $5 \mu \mathrm{g} \mathrm{mL}^{-1} \mathrm{ConA}$ as a positive control. For the sera samples, mice were bled $4 \mathrm{~h}$ after injection with SARS-CoV-2 LNP vaccine or control RABV vaccine and sera were collected. The cytokine response in each well was quantified with a custom 25-plex ProcartaPlex Immunoassay (ThermoFisher Scientific, UK) on a Bio-Plex 200 System (Bio-Rad), according to the manufacturer's instructions.

Statistical analysis. Graphs and statistics were prepared in GraphPad Prism (version 8.4). Statistical differences were analyzed using either a two-way ANOVA adjusted for multiple comparisons or a Kruskal-Wallis test adjusted for multiple comparisons, with $p<0.05$ used to indicate significance.

Reporting summary. Further information on research design is available in the Nature Research Reporting Summary linked to this article.

\section{Data availability}

The data that support the findings of this study are available within the article and its Supplementary Information files, or are available from the corresponding author upon reasonable request. Source data are provided with this paper.

Received: 2 June 2020; Accepted: 23 June 2020; Published online: 09 July 2020

\section{References}

1. Organization, W. H. Global COVID-19 statistics. https://covid19.who.int/ (2020).

2. Ksiazek, T. G. et al. A novel coronavirus associated with severe acute respiratory syndrome. N. Engl. J. Med. 348, 1953-1966 (2003). 
3. Zaki, A. M., van Boheemen, S., Bestebroer, T. M., Osterhaus, A. D. M. E. \& Fouchier, R. A. M. Isolation of a novel coronavirus from a man with pneumonia in Saudi Arabia. N. Engl. J. Med. 367, 1814-1820 (2012).

4. Al-Omari, A., Rabaan, A. A., Salih, S., Al-Tawfiq, J. A. \& Memish, Z. A. MERS coronavirus outbreak: Implications for emerging viral infections. Diagnostic Microbiol. Infect. Dis. 93, 265-285 (2019).

5. Kirchdoerfer, R. N. et al. Pre-fusion structure of a human coronavirus spike protein. Nature 531, 118-121 (2016).

6. Perri, S. et al. An alphavirus replicon particle chimera derived from venezuelan equine encephalitis and sindbis viruses is a potent gene-based vaccine delivery vector. J. Virol. 77, 10394-10403 (2003).

7. Blakney, A. K., McKay, P. F., Yus, B. I., Aldon, Y. \& Shattock, R. J. Inside out: optimization of lipid nanoparticle formulations for exterior complexation and in vivo delivery of saRNA. Gene Ther. 26, 363-372 (2019).

8. Blakney, A. K. et al. Big is Beautiful: Enhanced saRNA delivery and immunogenicity by a higher molecular weight, bioreducible, cationic polymer. ACS Nano14, 5711-5727 (2020).

9. Geall, A. J. et al. Nonviral delivery of self-amplifying RNA vaccines. Proc. Natl Acad. Sci. USA 109, 14604-14609 (2012).

10. Vogel, A. B. et al. Self-amplifying RNA vaccines give equivalent protection against influenza to mRNA vaccines but at much lower doses. Mol. Ther. 26, 446-455 (2018).

11. Garber, K. Alnylam launches era of RNAi drugs. Nat. Biotechnol. 36, 777-778 (2018).

12. Kim, E. et al. Microneedle array delivered recombinant coronavirus vaccines: Immunogenicity and rapid translational development. EBioMedicine 55, 102743 (2020)

13. Tetro, J. A. Is COVID-19 receiving ADE from other coronaviruses? Microbes Infect. 22, 72-73 (2020).

14. Wang, S.-F. et al. Antibody-dependent SARS coronavirus infection is mediated by antibodies against spike proteins. Biochem. Biophys. Res. Commun. 451, 208-214 (2014).

15. $\mathrm{Wu}, \mathrm{F}$. et al. A new coronavirus associated with human respiratory disease in China. Nature 579, 265-269 (2020).

16. Kirchdoerfer, R. N. et al. Stabilized coronavirus spikes are resistant to conformational changes induced by receptor recognition or proteolysis. Sci. Rep. 8, 15701-15701 (2018).

17. Pardi, N. et al. Expression kinetics of nucleoside-modified mRNA delivered in lipid nanoparticles to mice by various routes. J. Control. Release 217, 345-351 (2015).

18. Maier, M. A. et al. Biodegradable lipids enabling rapidly eliminated lipid nanoparticles for systemic delivery of RNAi therapeutics. Mol. Ther. 21, 1570-1578 (2013).

19. Jayaraman, M. et al. Maximizing the potency of siRNA lipid nanoparticles for hepatic gene silencing in vivo. Angew. Chem. 51, 8529-8533 (2012).

20. Badamchi-Zadeh, A. et al. Intramuscular immunisation with chlamydial proteins induces Chlamydia trachomatis specific ocular antibodies. PLoS ONE 10, e0141209 (2015).

21. Wright, E. et al. Investigating antibody neutralization of lyssaviruses using lentiviral pseudotypes: a cross-species comparison. J. Gen. Virol. 89, 2204-2213 (2008).

22. $\mathrm{Hu}, \mathrm{K}$. et al. CCL19 and CCL28 augment mucosal and systemic immune responses to HIV-1 gp140 by mobilizing responsive immunocytes into secondary lymph nodes and mucosal tissue. J. Immunol. 191, 1935 (2013).

\section{Acknowledgements}

We gratefully acknowledge Graham Taylor, Graham Cooke, Rachael Quinlan, Charlotte Short, and Carolina Rosadas de Oliveira for providing the samples from recovered
COVID-19 patients, and Jonathan Yeow for providing the polymers used in these studies. We thank Andrew Davidson and David Matthews, University of Bristol UK for supplying the VeroE6 cells used in this study. AKB is supported by a Marie Skłodowska Curie Individual Fellowship funded by the European Commission H2020 (No. 794059). P.F.M., K.H., K.S., C.R.B., K.P., P.R. and R.J.S. are funded by the Department of Health and Social Care using UK Aid funding and is managed by the Engineering and Physical Sciences Research Council (EPSRC, grant number: EP/R013764/1, note: the views expressed in this publication are those of the author(s) and not necessarily those of the Department of Health and Social Care). This work was supported in part by the NIHR Biomedical Research Centre of Imperial College Healthcare NHS Trust. We also acknowledge Dormeur Investment Services Ltd for providing funds to purchase equipment used in these studies.

\section{Author contributions}

P.F.M. and R.J.S. conceptualized the antigen design and designed the studies. P.F.M., K.S and K.H. designed and performed in vitro experiments. J.C.B., R.P., J.Z. and W.S.B. conceived of and performed in vitro wild type viral neutralization assays. P.F.M., A.K.B., K.H. and K.S. performed in vivo studies, aided by C.R.B., K.P., and P.R. P.J.C.L., C.B. and Y.K.T. designed and prepared the saRNA LNP. A.K.B. analysed the data and wrote the manuscript with help from P.F.M., K.H. and K.S., and constructive feedback and editing from R.P., J.B., C.R.B., K.P., P.R., W.B. and R.J.S.

\section{Competing interests}

P.F.M. and R.J.S. are co-inventors on a patent resulting from this work. P.J.C.L., C.B. and Y.K.T. are employed by Acuitas Therapeutics.

\section{Additional information}

Supplementary information is available for this paper at https://doi.org/10.1038/s41467 020-17409-9.

Correspondence and requests for materials should be addressed to R.J.S.

Peer review information Nature Communications thanks the anonymous reviewers for their contribution to the peer review of this work. Peer reviewer reports are available.

Reprints and permission information is available at http://www.nature.com/reprints

Publisher's note Springer Nature remains neutral with regard to jurisdictional claims in published maps and institutional affiliations.

Open Access This article is licensed under a Creative Commons Attribution 4.0 International License, which permits use, sharing, adaptation, distribution and reproduction in any medium or format, as long as you give appropriate credit to the original author(s) and the source, provide a link to the Creative Commons license, and indicate if changes were made. The images or other third party material in this article are included in the article's Creative Commons license, unless indicated otherwise in a credit line to the material. If material is not included in the article's Creative Commons license and your intended use is not permitted by statutory regulation or exceeds the permitted use, you will need to obtain permission directly from the copyright holder. To view a copy of this license, visit http://creativecommons.org/ licenses/by/4.0/

(C) The Author(s) 2020 